\title{
Individual differences in learning to perceive length by dynamic touch: Evidence for variation in perceptual learning capacities
}

\author{
Rob Withagen ANd MARgot VAN WermesKerken \\ University of Groningen, Groningen, The Netherlands
}

\begin{abstract}
Recent studies of perceptual learning have explored and commented on variation in learning trajectories. Although several factors have been suggested to account for this variation, thus far the idea that humans vary in their perceptual learning capacities has received scant attention. In the present experiment, we aimed at providing a detailed picture of the variation in this capacity by investigating the perceptual learning trajectories of a considerable number of participants. The learning process was studied using the paradigm of length perception by dynamic touch. The results showed that there are substantial individual differences in the way perceivers respond to feedback. Indeed, after feedback, the participants' perceptual performances diverged. We conclude that humans vary in their perceptual learning capacities. The implications of this finding for recent discussions on variation in perception are explored.
\end{abstract}

Most studies of perception assume a universal human nature. Indeed, some researchers assume that the perceptual system of the human species works in a certain way, and they try to discover the principles. The fact that the vast majority of studies do not examine individual performances but average over participants in their data analyses is indicative of this assumption. However, over the last decade or so, in several studies of perception, researchers have tried to reverse this trend. Indeed, these studies have explored and commented on individual differences in perception, often in the context of perceptual learning (e.g., Jacobs, Michaels, \& Runeson, 2000; Michaels \& de Vries, 1998; Runeson \& Andersson, 2007). Several of these studies adopted an ecological approach in order to examine these differences. This approach was introduced by James and Eleanor Gibson in the 1960s (E. J. Gibson, 1963; J. J. Gibson, 1966). Although both Gibsons worked on a theory of perceptual learning (J. J. Gibson \& E. J. Gibson, 1955), it was primarily developed by E. J. Gibson (1963). According to this ecological theory, perceptual learning is a process of differentiation: The perceiver learns to attend to the right informational variable in the ambient array. The underlying idea of this theory is that informational variables differ in degree of usefulness. Nonspecifying variables relate ambiguously to the to-be-perceived environmental property. That is, there is a many-to-many relationship between the environmental property and the informational variable. Hence, detecting such nonspecifying variables yields inaccuracies in the perceptual judgments of the property. Specifying variables, on the other hand, are variables that relate one-to-one to the environmental property and can therefore adequately constrain the perceptual judgment. Thus, perceivers can improve the accuracy of their perceptual judgments by learning to attend to the more useful information. This process has been termed the education of attention (E. J. Gibson, 1963; J. J. Gibson, 1966), or attunement, and has been demonstrated in several paradigms: the visual perception of the pulling force of a stick figure (e.g., Michaels \& de Vries, 1998), the visual perception of the relative mass of colliding balls (e.g., Jacobs et al., 2000; Jacobs, Runeson, \& Michaels, 2001; Runeson \& Andersson, 2007; Runeson, Juslin, \& Olsson, 2000), height and length perception by dynamic touch (Wagman, Shockley, Riley, \& Turvey, 2001; Withagen \& Michaels, 2005), visually guided braking (Fajen \& Devaney, 2006), and visually guided catching (van Hof, van der Kamp, \& Savelsbergh, 2006).

The ecologically inspired studies of perceptual learning focusing on individual differences have led to interesting discoveries (Jacobs et al., 2000; Jacobs et al., 2001; Michaels \& de Vries, 1998; Runeson \& Andersson, 2007; Runeson et al., 2000; Withagen \& Michaels, 2005). Indeed, individual differences have been revealed on at least two levels. First, participants were found to vary in the informational variables they initially relied on. Second, during the feedback phases of the experiments, the individual learning trajectories varied as well. That is, there was variation in the routes toward the mastery of the perceptual task. Some participants learned to exploit the more useful information after a relatively small number of feedback trials; others needed some more practice; and

R. G.Withagen, r.g.withagen@rug.nl 
some did not succeed in detecting that information during the experiment.

These demonstrations of variation are of interest for several reasons. First, as argued by Jacobs et al. (2000; Jacobs et al., 2001; see also Fajen, 2005), these findings cast serious doubt on the widely held assumption that all perceivers use the same informational variable to perform a (perceptual) task. Indeed, on the basis of a presumption of a universal human nature, many studies of perception and action assume that there is a single informational variable that is exploited by all members of the human species (for overviews, see Fajen, 2005; Jacobs \& Michaels, 2002; Tresilian, 1999). The finding that individuals vary in what informational variables they exploit, and that they can change in variable usage over time, suggests that this assumption is mistaken. Second, and related to the first reason, the demonstration of individual differences suggests that researchers of perception should shift focus. Instead of searching for a single informational variable that perceivers exploit in order to perform a task, perceptionists should examine the variation in what information is detected and try to explain it (Fajen, 2005; Jacobs et al., 2000; Jacobs et al., 2001; see also Cutting, 1986, 1991; Tresilian, 1999; van de Langenberg, Kingma, \& Beek, 2006; van der Kamp, Savelsbergh, \& Smeets, 1997). Indeed, the demonstration of individual differences raises several questions: How do we account for the between-subjects variation in learning paths? Why is there more variation in information usage in one experiment than in another? Why do perceivers learn to exploit the specifying information in one condition but not in another?

To date, several factors have been suggested to explain the observed variation in perceptual learning trajectories. For example, Jacobs et al. (2001) explored the relationship between the task ecology and the learning process. They contrived several task environments in which the usefulness of informational variables varied. If the informational variable that participants initially relied on had a high correlation with the to-be-perceived property, participants tended not to change in information usage after receiving feedback. When this correlation was rather weak, however, participants generally learned to detect the more useful information. Hence, Jacobs et al. (2001) concluded that whether perceptual learning occurs depends partly on the usefulness of the detected informational variables. In a similar vein, Fajen and Devaney (2006) explained variation in the learning process in terms of the range of practice conditions. When this range was limited, participants generally did not learn to exploit the specifying information. Participants did learn to detect the more useful information, however, when the range was extended. And recently, Runeson and Andersson (2007; see also Runeson et al., 2000) surmised the individual differences in the learning trajectories to be indicative of a chaotic search process among informational variables. ${ }^{1}$ That is, in achieving mastery of a perceptual skill, a perceiver tries different informational variables, which sooner or later results in the exploitation of the specifying information.
Up to this point, the idea that perceivers vary in their perceptual learning capacities has not gained serious attention in discussions on variation in learning. However, on the basis of an evolutionary analysis of perception and the use of information, Withagen and Chemero (in press) have argued that the evolutionary process probably gives rise to individual differences in perceptual systems. One of their main arguments is that in the course of evolution, natural selection is not likely to eliminate all available variation in perceptual systems in the evolving population. ${ }^{2}$ Hence, there is no such thing as the perceptual system of a species. Rather, the perceptual apparatuses of members of species vary. This means that perceivers are likely to vary in how well and how quickly they can learn a perceptual task. This idea of variation in perceptual learning capacities sheds some new light on recent findings and leads to new hypotheses. First, it provides an alternative explanation for the observed individual differences in learning trajectories. As previously mentioned, Runeson and Andersson (2007) surmised these differences to be attributable to a chaotic search that characterizes the perceptual learning process. However, the hypothesis that perceivers vary in their perceptual learning capacities also predicts individual differences in the mastery of a perceptual skill. After all, if animals vary in their learning capacities, they are likely to respond differently to the same feedback, implying between-subjects variation in the learning paths. Second, and related to this, the idea that animals vary in their perceptual learning capacities implies that providing feedback will not necessarily result in a convergence of the participants' perceptual performances. Indeed, one might even expect an increase in variation in what information is detected: Because of the individual differences in perceptual learning capacities, feedback will have a different effect on different participants, implying that their perceptual performances will diverge rather than converge.

Thus far, the ecologically inspired studies focusing on individual differences in perceptual learning trajectories have all run a limited number of participants in a particular condition $(8-10) .{ }^{3}$ Therefore, although there is evidence that the participants' perceptual learning paths vary, we still have only a rather limited insight into the nature and amount of variation in these paths. To judge whether this variation needs to be taken seriously in the theoretical and empirical study of perception, a more detailed portrayal of individual differences is needed. Hence, what is called for is a study of perceptual learning that uses a significantly larger sample size and tries to portray the individual differences in learning paths. The present experiment was designed to address those needs.

To study perceptual learning, we decided to use the paradigm of length perception by dynamic touch. In this paradigm, participants are asked to report the length of unseen, wielded rods. This perceptual task has proven to be a useful task for studying perceptual learning (Withagen \& Michaels, 2005; see also Wagman et al., 2001, for another study on learning in dynamic touch). Earlier studies have 
revealed that novice perceivers tend to rely on mechanical variables that correlate with rod length but are not specific to it. Indeed, at present, several nonspecifying mechanical variables have been implicated in length perception by dynamic touch. In the pioneering study of length perception by dynamic touch, the major principal moment of inertia $\left(I_{1}\right)$ was found to underlie length judgments (Solomon \& Turvey, 1988). In subsequent work, Fitzpatrick, Carello, and Turvey (1994) showed length perception by dynamic touch to be constrained by both $I_{1}$ and $I_{3}$, the minor principal moment of inertia. And recently, in a study by van de Langenberg et al. (2006; see also Kingma, Beek, \& van Dieën, 2002; Kingma, van de Langenberg, \& Beek, 2004), perceivers relied on $I_{1}$, the first moment of mass distribution $(M)$, and the zeroth moment of mass distribution $(m)$. More precisely, van de Langenberg et al. found that which mechanical variables their perceivers exploited depended on the mechanical context in which the length was to be judged.

In an environment consisting of homogeneous rods made of materials with different densities, all these mechanical variables relate ambiguously to the length of a rod. After all, these mechanical variables are all functions of the rod's length, radius, and material density. This means that rods of equal length can differ in $I_{1}, I_{3}, M$, and $m$, and that rods that are identical in these mechanical variables can differ in length. Therefore, several experimental studies have demonstrated that perceivers exploit nonspecifying variables when perceiving rod length.

Withagen and Michaels (2005), however, demonstrated that if feedback on the length estimations was provided, several participants learned to detect information that was more useful. The experimenters contrived a task ecology consisting of carbon pipes and homogeneous rods made of wood, aluminum, and steel, with several mechanical variables being specific to length. Consider, for instance, the ratio of the first moment of mass distribution to the zeroth moment of mass distribution,

$$
\frac{M}{m}=\frac{m \cdot L / 2}{m},
$$

where $m$ is mass and $L$ is length. Because mass cancels, the ratio of $M$ to $m$ is related one-to-one to rod length. That is, rods of the same length have the same $M / m$, even when the rods are made of materials with different densities. ${ }^{4}$ Withagen and Michaels found that if participants received feedback on their length judgments, several participants learned to detect a mechanical variable that specified length. Hence, given the general reliance on nonspecifying mechanical variables, and given the perceivers' capacity to come to rely on a specifying mechanical variable, the paradigm of length perception by dynamic touch is useful for studying perceptual learning.

\section{THE PRESENT EXPERIMENT}

The primary goal of the experiment was to portray individual differences in perceptual learning capacities. As previously mentioned, an evolutionary perspective on perception suggests that such differences are present in the current population. In the present experiment, the participants were trained to perceive the length of unseen wielded rods. Aiming at a detailed portrayal of the individual differences in learning, we used a relatively large sample size and provided each individual with a considerable number of feedback blocks. In these blocks, the participants received visual information about the length of the rod following their length estimation. To precisely keep track of changes in information usage, there was a test phase after each feedback block. To reveal whether attunement occurred and when, we tried to determine which mechanical variable constrained the participant's length judgments in each test phase.

\section{Method}

Participants. Twenty-five participants (13 women, 12 men) volunteered to participate by giving their informed consent. Their ages ranged from 18 to 26 years. Nineteen participants were righthanded; 6 were left-handed.

Apparatus and Materials. The apparatus was similar to those used in other studies of length perception by dynamic touch (see, e.g., Kingma et al., 2004; Solomon \& Turvey, 1988). The participant sat on a stool. In front of the participant, there was a rail with a small planar surface attached. The participant could move this surface along the rail by rotating a small wheel with the left hand. On the right side of the stool, there was an armrest supporting the participant's right forearm. Between the rail and the armrest, there was an opaque curtain that blocked the participant's vision, so that the wielded rod was not visible.

We used three distinct sets of rods. To prevent the participants from simply learning to identify individual rods, two sets of rods were used in the feedback phases, and one set was used in the test phases. Each set contained carbon pipes and uniform, solid rods made of steel, aluminum, or wood. By varying the diameters and the lengths of the rods, we contrived three distinct sets. Each rod had an $11.5-\mathrm{cm}$ plastic handle at one end, which was separated from the rod by a disk. This handle prevented the participant from feeling the material the rod was made of or its diameter.

The properties of the rods (both geometrical and mechanical) are provided in the Appendix. ${ }^{5}$ We were primarily concerned with the nonspecifying variables $I_{1}$ and $M$. These are the two mechanical variables that have been found to underlie length perception of horizontally wielded rods, at least when the variables are computed with respect to the end of the rod (Kingma et al., 2004; see note 5). For the test set, we chose the collection of rods so that $I_{1}$ and $M$ had low correlations with actual length (see Table 1). This means that the specifying and nonspecifying variables were disentangled, making it easier for us to determine whether the participants relied on a nonspecifying or a specifying informational variable. For the feedback sets, on the other hand, the collections of rods were chosen so that $I_{1}$ and $M$ had moderate correlations with actual length (see Table 1). We were primarily interested in the perceptual learning process in the animal's natural environment. It is our contention that in such an environment, there are often several informational variables that correlate moderately with, but are not specific to, the relevant environmental property (cf. Tresilian, 1999). Consequently, to warrant the ecological validity of the study, a moderate correlation between several nonspecifying variables and the to-be-perceived property is called for.

Procedure. The experiment consisted of seven test blocks and six feedback blocks, and was conducted over the course of 3 consecutive days. On each day, there were two feedback blocks, each of which was followed by a test block. To determine the participant's initial perceptual performance, there was an additional pretest on the first day. In each block, the participants were to estimate the length of the handheld rod. They were to position the planar surface so that it coincided with the felt maximum distance reachable with the 
Table 1

Correlations Between the Logarithms of the Candidate Variables and Actual Length

\begin{tabular}{|c|c|c|c|}
\hline & Length & $I_{1}$ & $M$ \\
\hline \multicolumn{4}{|c|}{ Feedback Rod Set 1} \\
\hline Length & - & .694 & .486 \\
\hline$I_{1}$ & - & - & .966 \\
\hline$M$ & - & - & - \\
\hline \multicolumn{4}{|c|}{ Feedback Rod Set 2} \\
\hline Length & - & .678 & .489 \\
\hline$I_{1}$ & - & - & .973 \\
\hline$M$ & - & - & - \\
\hline \multicolumn{4}{|c|}{ Test Rod Set } \\
\hline Length & - & .185 & -.138 \\
\hline$I_{1}$ & - & - & .948 \\
\hline$M$ & - & - & - \\
\hline
\end{tabular}

rod. Because a change in information usage was likely to be accompanied by a change in the wielding behavior (see Riley, Wagman, Santana, Carello, \& Turvey, 2002), the participants were allowed to wield the rod freely and to hold it loosely in their hand. The only restriction we imposed was that the participants were not allowed to touch the curtain or the floor.

The test phases consisted of 26 trials. Each test rod was offered twice, and the order of presentation was randomized. After each judgment, the participant was instructed to reposition the planar surface at the proximal end of the rail. The feedback blocks consisted of 24 trials. After the participants positioned the surface at the felt distance reachable, they were allowed to touch the curtain with the rod. This touching led to a curtain displacement that provided visual information about the position of the distal end of the handheld rod and, thus, about the accuracy of the perceptual judgment. Indeed, the distance between the position of the planar surface (the estimated distance reachable) and the curtain displacement (the actual distance reachable) informed the participants about the error in their perception of length. As in the test phases, the surface was to be repositioned at the proximal end of the rail after each trial. Half of the participants started each day with Feedback Set 1 and ended with Feedback Set 2 . The other half started with Feedback Set 2 followed by Feedback Set 1 . In each feedback phase, the rods were offered twice in a random order. Between the blocks, there was a small break.

Analyses. As mentioned earlier, the aim of the experiment was to examine individual differences in perceptual learning paths, so, contrary to most studies of perception, we did not average over participants in our analyses. Instead, we examined the perceptual performances of each individual for each test phase. To determine whether individuals changed the information they exploited, we adopted the method developed by Jacobs et al. (2000; see also Withagen \& Michaels, 2005, for an earlier exploitation of this method in the dynamic touch paradigm, which is identical to the one used here). For each individual and each test phase, we computed the Pearson product-moment correlations of perceived length with actual length and the nonspecifying variables $I_{1}$ and $M$. However, in computing the correlations, we used the logarithms of each of these variables. The reason was that the relationship between perceived length and the nonspecifying variables (most notably $I_{1}$ ) was expected to be nonlinear. To determine whether a participant had relied on a specifying or a nonspecifying variable, for each test phase, we compared the correlation of perceived length and actual length with the correlation of perceived length and the most highly correlated nonspecifying variable $\left(I_{1}\right.$ or $\left.M\right)$. We performed a $t$ test for dependent correlations (Bruning \& Kintz, 1987) to determine whether the difference between these two correlations was significant. A significant difference indicates that one informational variable explains the variance in perceived length significantly better than the other variable. Only when there was a significant difference between the two correlations did we conclude that the participant had relied on a specifying or a nonspecifying variable (depending on which of the correlations was higher).

\section{Results}

As expected, there were substantial individual differences in the learning trajectories. Although all but 3 participants (Participants 2, 7, and 19) relied on a nonspecifying variable in the pretest, their perceptual performances diverged over the course of the experiment. Participants varied in whether they responded to the feedback, when they responded to the feedback, how they responded to the feedback, whether they learned to detect the specifying information, how quickly they learned to detect this information, and how firmly they maintained reliance on informational variables. However, for the sake of clarity in the presentation of our findings, we have divided the participants into three groups: the learning group, the nolearning group, and the intermediate group.

The learning group consists of the participants who detected the specifying information in at least one test phase (see Figure 1). Although, strictly speaking, Participant 8 did not learn to detect the specifying information (i.e., the correlation of perceived length and actual length was never significantly higher than the correlation of perceived length and the most highly correlated nonspecifying variable), we assigned this participant to the learning group. This was done because Participant 8 showed considerable improvement in the length judgments - especially in comparison with the participants in the no-learning and intermediate groups. Although the remaining 4 participants all learned to exploit the specifying information, their learning paths showed considerable variation. For example, in line with earlier studies (Jacobs et al., 2000; Jacobs et al., 2001; Runeson \& Andersson, 2007; Withagen \& Michaels, 2005), the number of feedback trials that the participants needed in order to learn to exploit specifying information varied extensively. Participant 2 was the quickest learner, followed by Participants 1, 10, and 19. Furthermore, and also in keeping with the aforementioned studies, we found that the discovery of specifying information did not guarantee that participants would rely on this information. For example, Participant 10 detected the specifying information in the fifth test phase but did not stick to this variable in subsequent test blocks. Also, Participants 1, 2 , and 8 showed drops in the accuracy of their perceptual performances after the specifying variable was discovered. Apparently, the perceptual learning process does not consist in a progressive convergence on the specifying information.

The no-learning group comprised 10 participants (see Figure 2). These participants generally relied on a nonspecifying variable during the experiment, and feedback did not seem to have any effect on these participants. The fact that a substantial number of participants were almost unaffected by the feedback suggests that the no-learners 


\section{Learning Group}

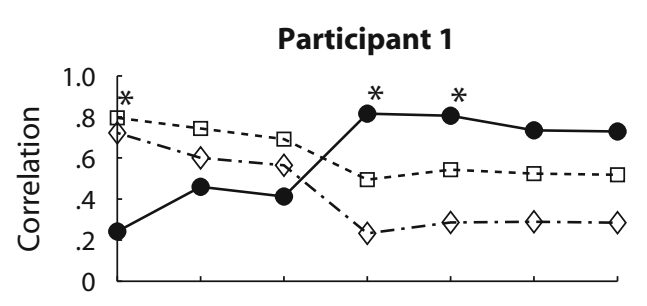

Participant 8
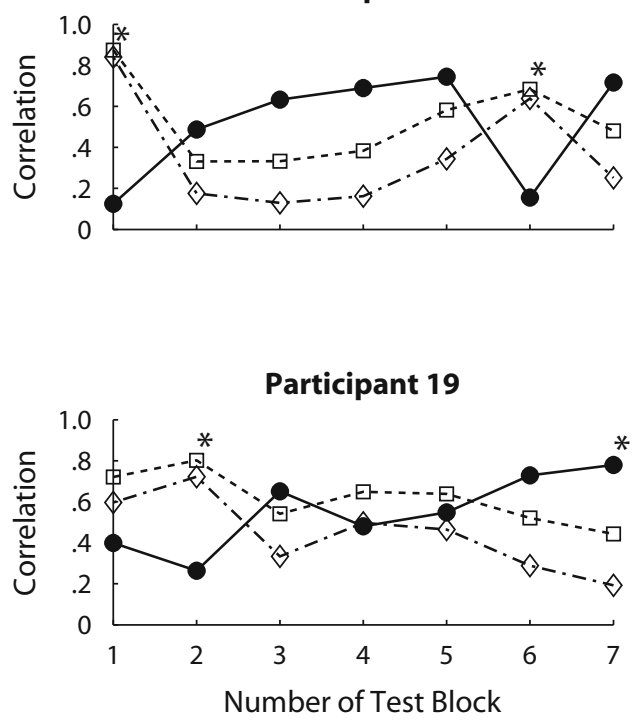

Participant 2

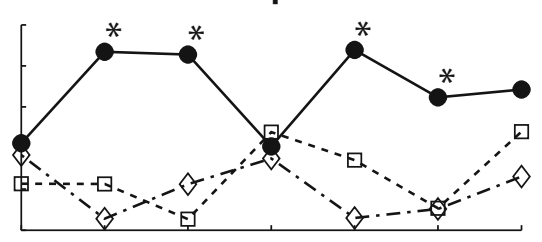

Participant 10

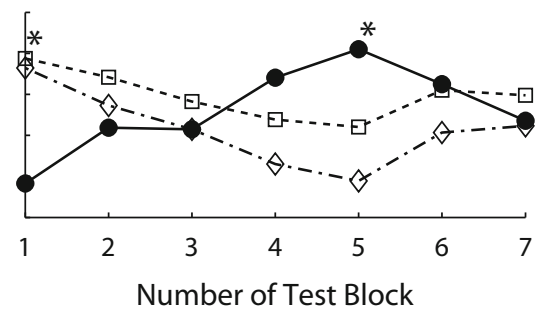

Length

- - $---I_{1}$

$-\vartheta \cdot-M$

Figure 1. The learning group's correlations between the length judgments and $I_{1}, M$, and actual length in the test phases. In the blocks marked with an asterisk, there is a significant difference $(p<$ .05 , two tailed) between the correlation of perceived length with actual length and the correlation of perceived length with the most highly correlated nonspecifying variable.

observed in earlier studies (e.g., Jacobs et al., 2000; Jacobs et al., 2001; Michaels \& de Vries, 1998; Runeson \& Andersson, 2007; Withagen \& Michaels, 2005) were not simply outliers. Indeed, our results indicate that a significant part of the population maintains reliance on nonspecifying information when this allows moderate perceptual performance.

The 10 participants in the intermediate group were affected by the feedback, but they did not succeed in learning to detect the specifying information (see Figure 3). Despite this common factor, there was substantial between-subjects variation in this group. First, participants varied in when they responded to the feedback. The perceptual performances of Participants 5, 7, and 21 were clearly affected by the first feedback block. Participants $6,14,16$, and 22 , on the other hand, maintained reliance on a nonspecifying variable in the first phases of the experiment. Apparently, participants varied in how quickly they were able to take advantage of feedback information. Second, there was variation in how participants responded to feedback. For example, Participant 7 learned to exploit a nonspecifying variable in the first feedback block. Other participants (Participants 5 and 21) learned not to exploit such information in that feedback session. Furthermore, Participant 22 returned to the use of a nonspecifying variable after having failed to discover the specifying information. Participants $6,7,12,14,17,21$, and 25 , on the other hand, did not demonstrate this return to the variable that they had initially exploited. Indeed, these participants had relatively low correlations with actual length and the nonspecifying variables $I_{1}$ and $M$ in the second half of the experiment. This may indicate that these participants switched between these variables in later test blocks, or that they relied on an informational variable that we did not consider.

In summary, we conclude that there are strong individual differences in the learning paths. The fact that the participants' perceptual performances diverged after feedback suggests that their perceptual learning capacities varied.

\section{DISCUSSION}

In the experiment reported here, perceivers were trained to estimate the length of unseen wielded rods. Using a relatively large sample size, we aimed at gaining insight into 


\section{No-Learning Group}

\section{Participant 3}

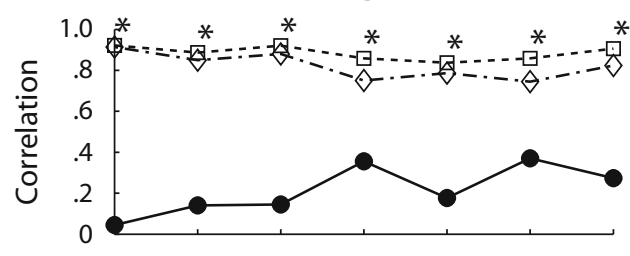

\section{Participant 9}

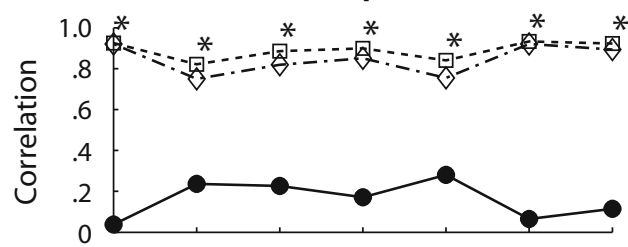

Participant 13

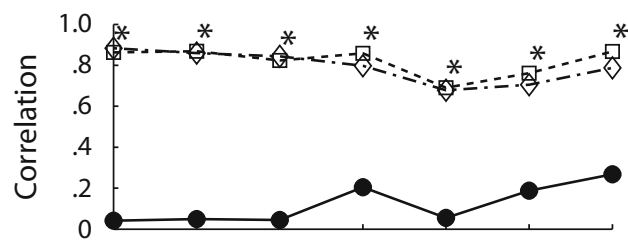

Participant 18

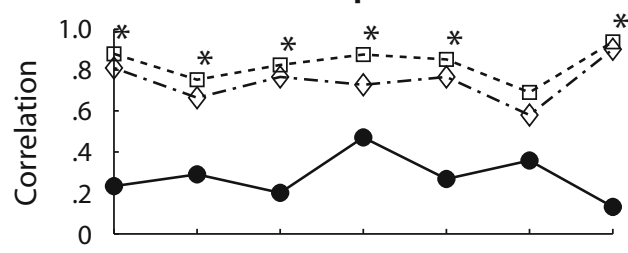

Participant 23

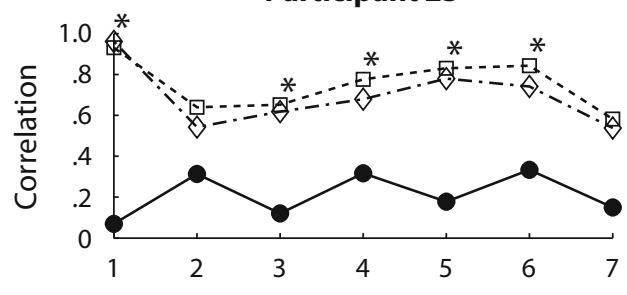

Number of Test Block

\section{Participant 4}

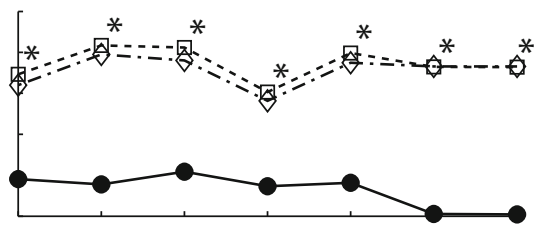

Participant 11

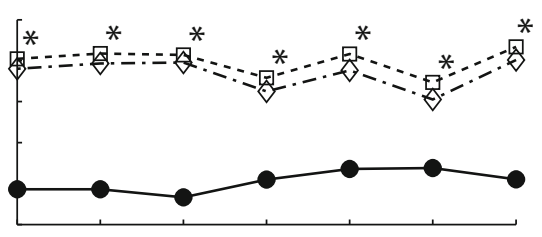

Participant 15

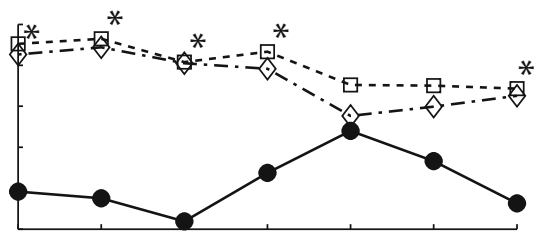

Participant 20

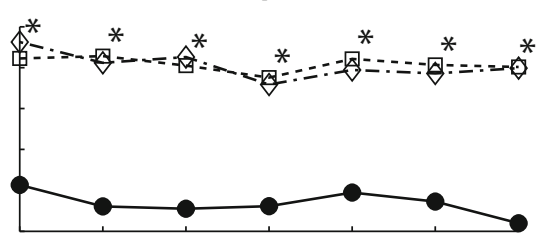

Participant 24

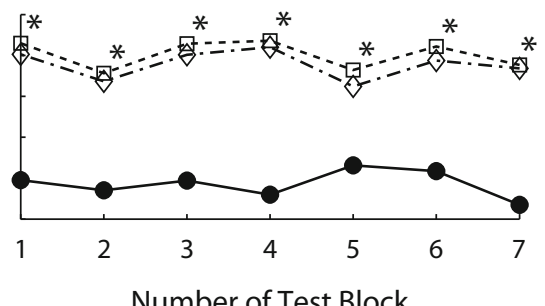

$$
\begin{array}{ll}
\longrightarrow-\text { Length } \\
--\square--I_{1} \\
-\backsim \cdot-M
\end{array}
$$

Figure 2. The no-learning group's correlations between the length judgments and $I_{1}, M$, and actual length in the test phases. In the blocks marked with an asterisk, there is a significant difference $(p<$ .05 , two tailed) between the correlation of perceived length with actual length and the correlation of perceived length with the most highly correlated nonspecifying variable. 


\section{Intermediate Group}
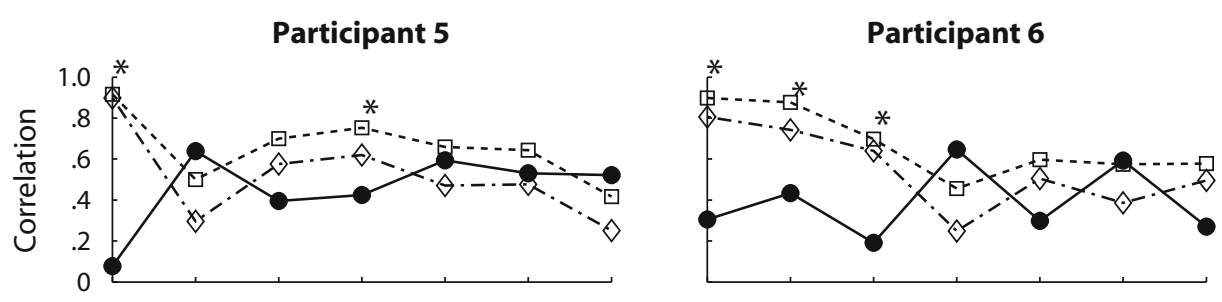

Participant 7

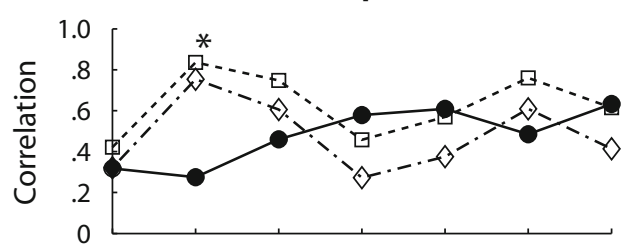

Participant 12

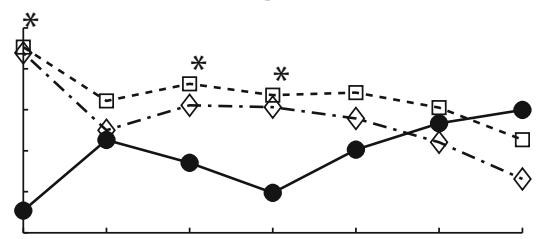

Participant 14

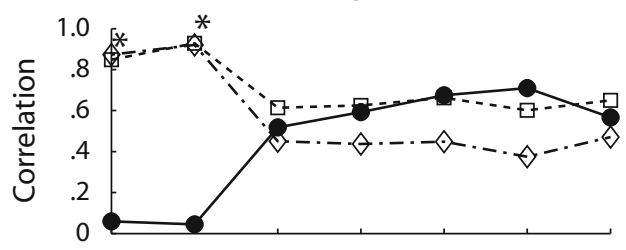

Participant 16

Participant 17

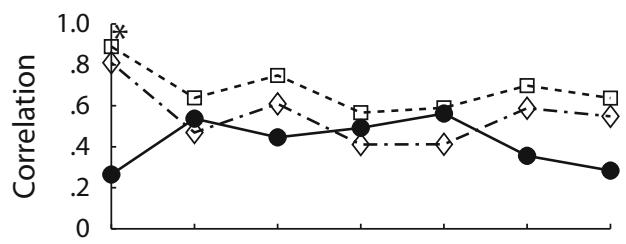

Participant 21

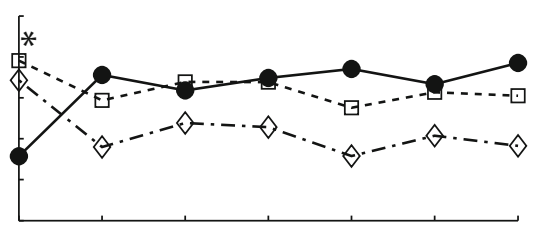

Participant 22

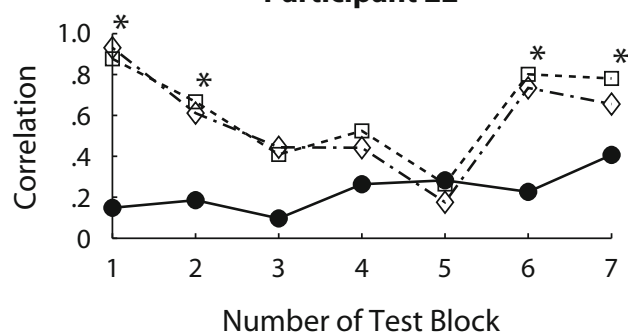

Participant 25

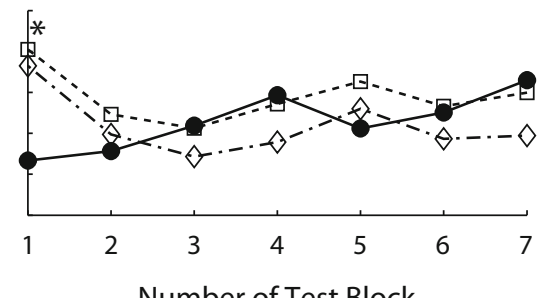

$$
\begin{aligned}
& \longrightarrow-\text { Length } \\
& --\square--I_{1} \\
& -\diamond \cdot-M
\end{aligned}
$$

Figure 3. The intermediate group's correlations between the length judgments and $I_{1}, M$, and actual length in the test phases. In the blocks marked with an asterisk, there is a significant difference $(p<.05$, two tailed) between the correlation of perceived length with actual length and the correlation of perceived length with the most highly correlated nonspecifying variable. 
the nature and amount of individual differences in perceptual learning capacities. The experiment was motivated by a theoretical study that suggested that from an evolutionary perspective, members of a species are likely to vary in this capacity. We indeed found substantial betweensubjects variation in the learning trajectories. Although at a fine grain of analysis the participant's paths were unique with respect to each other, we divided the participants into three groups. Forty percent of the participants proved not to be sensitive to the feedback and generally relied on nonspecifying information during the whole experiment. Another $40 \%$ of the participants changed their perceptual judgments after being provided feedback, but they did not succeed in discovering the specifying information. And $20 \%$ of the participants learned to detect the specifiying information over the course of the experiment. We took this variation in the learning paths as evidence for individual differences in perceptual learning capacities.

In what follows, we discuss the implications of our findings in relation to recent discussions on variation in perception. We conclude with speculations on perceptual learning capacities.

\section{Individual Differences in Perception}

As mentioned in the introduction, several recent studies of perceptual learning have already emphasized individual differences in perception (e.g., Jacobs et al., 2000; Jacobs et al., 2001; Michaels \& de Vries, 1998; Runeson \& Andersson, 2007; Runeson et al., 2000). Contrary to a still dominant assumption in the study of perception and action, these studies have suggested that there is no such thing as the informational variable that participants use to perform a certain (perceptual) task. Rather, members of species vary in the information that they detect. Furthermore, perceivers often change the information they use over time.

Although the present study is nothing more than a detailed portrayal of the individual differences in perceptual learning paths, it has, we believe, important implications for the discussion of individual differences in perception. Among other things, the study provides another argument for taking individual differences seriously in the theoretical and empirical study of perception. Earlier studies of perceptual learning have sometimes concluded that perceivers may initially vary in the information they exploit, but that perceivers will generally converge on the more useful informational variables after feedback (see, e.g., Jacobs \& Michaels, 2002). Hence, they expect that between-subjects variation in the information that is detected occurs primarily over the short term. The present study casts doubt on this idea. All but 3 participants relied on a nonspecifying variable in the pretest. However, after feedback, their perceptual performances diverged. The fact that a substantial number of participants were almost unaffected by the feedback suggests that a general convergence on the specifying information would not have occurred even if more feedback had been provided. This indicates that individual differences in the information that is detected are not confined to the short term. Rather, also as on the long term (i.e., in everyday perceptual tasks), it is quite likely that different members of a species will rely on different informational variables.

\section{Chaotic Search Process, Individual Differences in Motivation, or Variation in Perceptual Learning Capacities?}

We interpret the individual differences in the learning paths to be evidence of variation in perceptual learning capacities. But are there other explanations for our results? In this section, we explore two alternative explanations for our findings: Runeson's chaotic search hypothesis and the idea that humans are differently motivated to learn a perceptual task.

As mentioned in the introduction, Runeson and Andersson (2007; see also Runeson et al., 2000) suggested that individual differences in the mastery of a perceptual task are indicative of a chaotic search among informational variables. Can this hypothesis also account for our data? Although some of our results could also be explained in terms of a chaotic search process, we believe that this hypothesis cannot account for some of our important observations. Runeson's hypothesis of a chaotic search is a statement about the nature of the perceptual learning process: The perceiver will simply try different variables and might sooner or later discover the more useful information. Hence, the chaotic search hypothesis predicts variation in how perceivers respond to feedback. This means that the different paths of the participants in the learning group can be accounted for in terms of a chaotic search process. In addition, the fact that the 10 participants in the intermediate group did not succeed in discovering the specifying information could also be indicative of a chaotic search process. After all, such a process does not guarantee that the perceiver will ever detect the specifying information. Indeed, the nature of this process implies that whether this information will be discovered is basically a matter of chance. However, Runeson's hypothesis of a chaotic search cannot account for the fact that our participants varied in whether and when they responded to the feedback. Indeed, the demonstration that a substantial number of participants were unaffected by the feedback is not attributable to a chaotic search process among variables. This also holds true for the fact that participants varied in when they responded to feedback.

One could argue that the variation in whether and when participants responded to feedback was the result of individual differences in motivation. After all, motivation to learn a perceptual task is likely to facilitate the process of attunement. Motivation may well be a necessary prerequisite for perceptual learning to occur. We believe that the observed individual differences in learning trajectories were partly attributable to between-subjects variation in motivation. Humans do vary in many dimensions, and motivation to learn and perform a perceptual task is certainly one of them. However, the omnipresent variation in the human species also implies that it is unlikely that the individual differences in the learning paths are exclusively the result of differently motivated participants. Indeed, 
as mentioned in the introduction, an earlier evolutionary analysis of perception suggested that perceivers vary in how well and how quickly they can learn a perceptual task. The individual differences in the learning trajectories are also likely to reflect this variation. Indeed, the differences in whether and when participants responded to the feedback indicate that perceivers vary in their capacity to take advantage of feedback information. Hence, although we recognize that participants are differently motivated to learn the task, we believe that the observed betweensubjects variation in the learning trajactories provides evidence for the idea that members of a species vary in their perceptual learning capacities.

\section{How to Account for Variation \\ in the Information That Is Detected}

The present study also has implications for the discussion about how to explain variation in what information is detected. In fact, the study adds another determinant to the growing list of factors that have been implicated in determining what information is detected. Earlier studies of variation in information usage focused primarily on environmental factors. In the growing body of work on perceptual learning (Fajen \& Devaney, 2006; Jacobs et al., 2000; Jacobs et al., 2001; Michaels \& de Vries, 1998; Runeson \& Andersson, 2007; Runeson et al., 2000; Wagman et al., 2001), feedback has been revealed to be an important factor that induces changes in information usage over time. In fact, Jacobs and Michaels (2007) recently developed a rather sophisticated theoretical framework that aims at capturing how feedback guides a perceiver through an information space. And in line with Cutting's $(1986,1991)$ directed perception theory, several studies have shown that what information is detected depends on the environmental context. For example, and as mentioned in the introduction, van de Langenberg et al. (2006) showed that which mechanical variables constrain length perception by dynamic touch depends on the mechanical context in which the task has to be performed (for other examples, see Cutting, 1991; Tresilian, 1999).

The present study shows that besides environmental factors, there is also an organismal factor involved in determining what information is detected. Participants varied in whether, when, and how they responded to the feedback. Again, this indicates that animals vary in their perceptual learning capacities. This conclusion has several implications. Importantly, it questions the assumption of a universal human nature that underlies most studies of perception. ${ }^{6}$ Even studies of variation in what information is exploited have assumed a universal perceptual mechanism. For example, several studies have tried to reveal (lawful) relationships between environmental conditions and what information is exploited (see, e.g., Cutting, 1991; Tresilian, 1999). However, in doing so, they have assumed that all members of a species act the same in the same environmental context. Revealing substantial individual differences in the learning paths, the present study, however, suggests that the perceptual apparatuses of members of a species vary.
It is important to note that this variation does not imply that a scientific theory of the use of informational variables is impossible. The fact that animals are unique does not mean that one should formulate a theory for each and every individual, rendering any generalization impossible. Rather, the individual differences suggest that in explaining variation in what information is used, one should incorporate organismal factors as well. In fact, the ongoing discovery of factors that determine information usage indicates that, as with other variable organismal features (e.g., height, weight, intelligence, development), the use of information is under the influence of several environmental and organismal factors. Hence, a theory of the detection of information should explain how the interplay of these factors determines what information is relied on (Withagen \& Chemero, in press).

At present, we are far from this type of naturalistic theory of the use of information. Presumably, we have not revealed all of the factors that are involved. Indeed, we have only begun scrutinizing factors that possibly determine what information is exploited. For example, as previously mentioned, participants are likely to vary in their motivation to learn and perform a task, and the effects of this variation on what information is exploited needs to be examined. In addition, it is not unlikely that the observed variation in the learning trajectories is partly influenced by genetic factors. Earlier studies have revealed that there is a genetic component underlying normal variation in several psychological traits (see, e.g., Fox, Hershberger, \& Bouchard, 1996; Plomin, 1990; Ramus, 2006). And recent work on cultural differences in perception and attention suggests that there is also a cultural component to the determination of what information is used (see, e.g., Nisbett, 2003; Nisbett \& Miyamoto, 2005). Furthermore, substantial work is needed to develop a theoretical framework that is capable of explaining how multiple organismal and environmental factors jointly determine what information is exploited. However, to our minds, developmental systems theory is a very promising approach in developing such an account. According to this theory, organismal features, including (perceptual) skills, evolve through the mutual interaction of several factors (see, e.g., Ingold, 2000; Oyama, 1985, 2000; Oyama, Griffiths, \& Gray, 2001; Thelen \& Smith, 1994). Hence, it is the entire system of interactions that needs to be taken into account in the understanding of the development of a skill. Applying the principles of developmental systems theory to the study of perceptual learning is, we believe, likely to help in the development of an account of how the interplay of factors determines what information is detected.

\section{Perceptual Learning Capacities}

Although we have been discussing the fact that participants vary in their perceptual learning capacities, we have not been very precise about what this variation comprises. In the remainder of this article, we will speculate about individual differences in this capacity.

As touched on earlier, one feature that perceivers might vary in is the ability to take advantage of feed- 
back information. From probability judgment research, Runeson and colleagues adopted the distinction between Thurstonian errors and Brunswikian errors (Runeson \& Andersson, 2007; Runeson et al., 2000). Brunswikian errors are the result of the detection of nonspecifying informational variables. Thurstonian errors, on the other hand, are the result of noise in the perceptual system. Hence, for feedback to induce a change in what information is used, perceivers should be capable of distinguishing Thurstonian from Brunswikian errors. That is, they should be capable of separating the noise from the error that is the result of the detection of nonspecifying information.

It is not unlikely that perceivers vary in their capacity to distinguish these two types of error. Indeed, variation in this capacity can account for the fact that participants varied in whether they responded to feedback. For example, participants in the no-learning group might not have been capable of detecting the Brunswikian errors. Consequently, they maintained reliance on nonspecifying information over the course of the experiment. The participants in the intermediate and learning groups, on the other hand, were affected by the feedback. Hence, they perceived the fedback error not as noise, but as the result of the exploitation of a nonspecifying variable. As a result, they changed in information usage. One could argue that the better a perceiver is at detecting Brunswikian errors, the smaller the number of feedback trials that are needed to induce a change in information usage. This means that variation in this capacity could also explain the observed individual differences in when feedback resulted in a change. That is, the participants who were affected by the first feedback phase were likely to be better at detecting Brunswikian errors than were the participants who needed more feedback.

At present, it is unclear whether variation in the capacity for taking advantage of feedback can also explain the differences in how perceivers respond to feedback. Whether it can depends at least partly on the nature of the learning process. In the ecological literature on perceptual learning, there is some controversy on this topic. Runeson and colleagues (Runeson \& Andersson, 2007; Runeson et al., 2000) have suggested that learning includes a chaotic search from among variables. Hence, a well-developed capacity to detect Brunswikian errors means that such a search is often initiated. However, the route to the mastery of the skill is chaotic and thus not predictable. Jacobs and Michaels (2007), on the other hand, have suggested that there is no chaotic search among informational variables. Instead, feedback guides a perceiver systematically to the more useful informational variable. This would mean that variation in the capacity for taking advantage of feedback could also explain the differences in how perceivers respond to feedback. For example, a perceiver who is skilled in doing so is likely to achieve the detection of specifying information rather quickly.

The studies to date, however, do not equip us to decide between these two views of the perceptual learning process. The observation that several perceivers progressively converge on specifying information is in favor of Jacobs and Michaels's (2007) theory and casts doubt on the chaotic search hypothesis. However, the oft-reported fluctuations in what information is exploited provide evidence for Runeson's hypothesis and question the idea that feedback guides a perceiver systematically to the more useful information. Hence, to settle this issue and, more generally, to develop a fully fledged theory of attunement, further research is required. The present study shows that in developing this theory, individual differences in the learning process need to be recognized. The variation in whether, when, and how perceivers respond to feedback appears to be ubiquitous.

\section{AUTHOR NOTE}

Margot van Wermeskerken is now at Research Institute MOVE, Faculty of Human Movement Sciences, Vrije University Amsterdam, Amsterdam, The Netherlands. We are grateful to John van der Kamp and two anonymous reviewers for useful comments on an earlier version of the article. Correspondence concerning this article and reprint requests should be addressed to R. Withagen, Center for Human Movement Sciences, University of Groningen, P.O. Box 196, 9700 AD Groningen, The Netherlands (e-mail: r.g.withagen@rug.nl).

\section{REFERENCES}

BRUning, J. L., \& Kintz, B. L. (1987). Computational handbook of statistics (3rd ed.). Glenview, IL: Scott Foresman.

Cutting, J. E. (1986). Perception with an eye for motion. Cambridge, MA: MIT Press.

CutTing, J. E. (1991). Four ways to reject directed perception. Ecological Psychology, 3, 25-34.

FAJEN, B. R. (2005). Perceiving possibilities for action: On the necessity of calibration and perceptual learning for the visual guidance of action. Perception, 34, 717-740.

FAJEN, B. R., \& DeVANEY, M. C. (2006). Learning to control collisions: The role of perceptual attunement and action boundaries. Journal of Experimental Psychology: Human Perception \& Performance, 32, 300-313.

FitzPatrick, P., CARello, C., \& Turvey, M. T. (1994). Eigenvalues of the inertia tensor and exteroception by the "muscle sense." Neuroscience, 60, 551-568.

Fox, P. W., Hershberger, S. L., \& Bouchard, T. J., JR. (1996). Genetic and environmental contributions to the acquisition of a motor skill. Nature, 384, 356-358.

Gibson, E. J. (1963). Perceptual learning. Annual Review of Psychology, 14, 29-56.

GiBson, J. J. (1966). The senses considered as perceptual systems. Boston: Houghton Mifflin.

Gibson, J. J., \& Gibson, E. J. (1955). Perceptual learning: Differentiation or enrichment? Psychological Review, 62, 32-41.

INGold, T. (2000). Evolving skills. In H. Rose \& S. Rose (Eds.), Alas, poor Darwin: Arguments against evolutionary psychology (pp. 273298). New York: Harmony.

INGOLD, T. (2004). Beyond biology and culture: The meaning of evolution in a relational world. Social Anthropology, 12, 209-221.

JaCoBs, D. M., \& Michaels, C. F. (2002). On the apparent paradox of learning and realism. Ecological Psychology, 14, 127-139.

JaCOBS, D. M., \& Michaels, C. F. (2007). Direct learning. Ecological Psychology, 19, 321-349.

Jacobs, D. M., Michaels, C. F., \& Runeson, S. (2000). Learning to perceive the relative mass of colliding balls: The effects of ratio scaling and feedback. Perception \& Psychophysics, 62, 1332-1340.

Jacobs, D. M., Runeson, S., \& Michaels, C. F. (2001). Learning to visually perceive the relative mass of colliding balls in globally and locally constrained task ecologies. Journal of Experimental Psychology: Human Perception \& Performance, 27, 1019-1038.

Kingma, I., BeeK, P. J., \& van DieËN, J. H. (2002). The inertia tensor versus static moment and mass in perceiving length and heaviness of hand-wielded rods. Journal of Experimental Psychology: Human Perception \& Performance, 28, 180-191.

Kingma, I., van de Langenberg, R., \& Beek, P. J. (2004). Which mechanical invariants are associated with the perception of length 
and heaviness of a nonvisible handheld rod? Testing the inertia tensor hypothesis. Journal of Experimental Psychology: Human Perception \& Performance, 30, 346-354.

LoOren de Jong, H., \& VAN DER STEen, W. J. (1998). Biological thinking in evolutionary psychology: Rockbottom or quicksand? Philosophical Psychology, 11, 183-205.

Michaels, C. F., \& DE VRies, M. M. (1998). Higher order and lower order variables in the visual perception of relative pulling force. Journal of Experimental Psychology: Human Perception \& Performance, 24, 526-546.

NisBetT, R. E. (2003). The geography of thought: How Asians and Westerners think differently ... and why. New York: Free Press.

Nisbetт, R. E., \& Miyamoto, Y. (2005). The influence of culture: Holistic versus analytic perception. Trends in Cognitive Sciences, $\mathbf{9}$, 467-473.

Oyama, S. (1985). The ontogeny of information: Developmental systems and evolution. New York: Cambridge University Press.

Oyama, S. (2000). Evolution's eye: A systems view of the biologyculture divide. Durham, NC: Duke University Press.

Oyama, S., Griffiths, P. E., \& Gray, R. D. (Eds.) (2001). Cycles of contingency: Developmental systems and evolution. Cambridge, MA: MIT Press.

Plomin, R. (1990). The role of inheritance in behavior. Science, $\mathbf{2 4 8 ,}$ 183-188.

Ramus, F. (2006). Genes, brain, and cognition: A roadmap for the cognitive scientist. Cognition, 101, 247-269.

Riley, M. A., Wagman, J. B., Santana, M.-V., Carello, C., \& TurVEY, M. T. (2002). Perceptual behavior: Recurrence analysis of a haptic exploratory procedure. Perception, 31, 481-510.

Runeson, S., \& ANDERsson, I. E. K. (2007). Achievement of specificational information usage with true and false feedback in learning a visual relative-mass discrimination task. Journal of Experimental Psychology: Human Perception \& Performance, 33, 163-182.

Runeson, S., Juslin, P., \& Olsson, H. (2000). Visual perception of dynamic properties: Cue heuristics versus direct-perceptual competence. Psychological Review, 107, 525-555.

Solomon, H. Y., \& TurveY, M. T. (1988). Haptically perceiving the distances reachable with hand-held objects. Journal of Experimental Psychology: Human Perception \& Performance, 14, 404-427.

Thelen, E., \& Sмith, L. B. (1994). A dynamic systems approach to the development of cognition and action. Cambridge, MA: MIT Press.

Tоову, J., \& Cosmides, L. (1990). On the universality of human nature and the uniqueness of the individual: The role of genetics and adaptation. Journal of Personality, 58, 17-67.

Tresilian, J. R. (1999). Visually timed action: Time-out for 'tau'? Trends in Cognitive Sciences, 3, 301-310.

van de Langenberg, R., Kingma, I., \& Beek, P. J. (2006). Mechanical invariants are implicated in dynamic touch as a function of their salience in the stimulus flow. Journal of Experimental Psychology: Human Perception \& Performance, 32, 1093-1106.

van der Kamp, J., Savelsbergh, G., \& Smeets, J. (1997). Multiple information sources in interceptive timing. Human Movement Science, 16, 787-821.

van Hof, P., van Der Kamp, J., \& Savelsbergh, G. J. P. (2006). Threeto eight-month-old infants' catching under monocular and binocular vision. Human Movement Science, 25, 18-36.

Wagman, J. B., Shockley, K., Riley, M. A., \& Turvey, M. T. (2001).
Attunement, calibration, and exploration in fast haptic perceptual learning. Journal of Motor Behavior, 33, 323-327.

WILSON, D. S. (1994). Adaptive genetic variation and human evolutionary psychology. Ethology \& Sociobiology, 15, 219-235.

Withagen, R., \& Chemero, A. (in press). Naturalizing perception: Developing the Gibsonian approach to perception along evolutionary lines. Theory \& Psychology.

Withagen, R., \& Michaels, C. F. (2005). The role of feedback information for calibration and attunement in perceiving length by dynamic touch. Journal of Experimental Psychology: Human Perception \& Performance, 31, 1379-1390.

\section{NOTES}

1. It is important to note that Runeson and colleagues introduced the chaotic search hypothesis because they considered it a plausible theory of learning that not only can account for individual differences but also can explain the individual's fluctuations in variable usage.

2. It is beyond the scope of the article to completely spell out Withagen and Chemero's (in press) argumentation. Hence, we refer the reader who is interested in the theoretical background of this study to their paper.

3. It is important to note that Fajen and Devaney (2006) and Runeson et al. (2000) used larger sample sizes (15-20). However, Fajen and Devaney did not focus on individual differences in the learning trajectories. And Runeson et al.'s study was limited in tracking the variable usage of individuals, as Runeson himself acknowledged (Runeson \& Andersson, 2007).

4. Again, it is important to note that in an environment consisting of homogeneous rods, this variable is just one example of a mechanical variable that specifies length. Indeed, any ratio of two moments of mass distribution is specific to the length of homogeneous rods. After all, the prerequisite is that the mass cancels out, leaving the mechanical variable a single-valued function of rod length.

5. It is important to point out that we computed the moments of mass distribution with respect to the end of the rod. In the literature on dynamic touch, there is discussion on how to compute the mechanical variables. Turvey, Carello, and colleagues have computed the moments of inertia with respect to the wrist (e.g., Fitzpatrick et al., 1994; Solomon \& Turvey, 1988). Van de Langenberg et al. (2006), on the other hand, argued that the moments of mass distribution ought to be computed with respect to the end of the rod. For reasons spelled out in the Method section, the participants in the present study were allowed to hold the rods loosely in their hand. This means that the distance between the proximal end of the rod and the wrist was likely to vary over the course of the experiment. Hence, we decided to compute the moments of mass distribution with respect to the more neutral point, the end of the rod. However, mechanical variables differ when computed with respect to different loci, rendering it difficult to compare the results of the different studies (cf. Withagen \& Michaels, 2005 , footnote 3 ). Consequently, the decision concerning which informational variables to consider in our analyses is based on studies that take the end of the rod as the reference point.

6. In fact, this assumption is dominant in psychology. Even some prominent evolutionary psychologists have argued for a universal human nature (e.g., Tooby \& Cosmides, 1990). However, this view has not gone unchallenged. Recently, scientists from different academic disciplines have argued against it (e.g., Ingold, 2004; Looren de Jong \& van der Steen, 1998; Nisbett, 2003; Wilson, 1994). 


\section{APPENDIX}

Properties of the Rods Used in the Feedback and Test Blocks

\begin{tabular}{|c|c|c|c|c|c|c|c|}
\hline & Material & $\begin{array}{l}\text { Length } \\
\text { (m) }\end{array}$ & $\begin{array}{l}\text { Diameter } \\
(\mathrm{m})\end{array}$ & $\begin{array}{c}m \\
(\mathrm{~kg})\end{array}$ & $\begin{array}{c}M \\
(\mathrm{~kg} \cdot \mathrm{m})\end{array}$ & $\begin{array}{c}I_{1} \\
\left(\mathrm{~kg} \cdot \mathrm{m}^{2}\right)\end{array}$ & $\begin{array}{c}I_{3} \cdot 10^{4} \\
\left(\mathrm{~kg} \cdot \mathrm{m}^{2}\right)\end{array}$ \\
\hline \multicolumn{8}{|c|}{ Feedback Rod Set 1} \\
\hline 1 & aluminum & 0.60 & 0.010 & 0.127 & 0.038 & 0.015 & 0.016 \\
\hline 2 & aluminum & 0.70 & 0.010 & 0.150 & 0.053 & 0.025 & 0.019 \\
\hline 3 & steel & 0.30 & 0.010 & 0.173 & 0.026 & 0.005 & 0.022 \\
\hline 4 & steel & 0.50 & 0.010 & 0.309 & 0.077 & 0.026 & 0.039 \\
\hline 5 & steel & 0.70 & 0.010 & 0.432 & 0.151 & 0.071 & 0.054 \\
\hline 6 & wood & 0.60 & 0.012 & 0.044 & 0.013 & 0.005 & 0.008 \\
\hline 7 & aluminum & 0.60 & 0.014 & 0.249 & 0.075 & 0.030 & 0.061 \\
\hline 8 & aluminum & 0.40 & 0.016 & 0.215 & 0.043 & 0.011 & 0.069 \\
\hline 9 & aluminum & 0.70 & 0.016 & 0.384 & 0.135 & 0.063 & 0.123 \\
\hline 10 & aluminum & 0.80 & 0.016 & 0.438 & 0.175 & 0.094 & 0.140 \\
\hline 11 & carbon & 0.50 & 0.020 & 0.064 & 0.016 & 0.005 & 0.055 \\
\hline 12 & carbon & 0.80 & 0.020 & 0.103 & 0.041 & 0.022 & 0.089 \\
\hline \multicolumn{8}{|c|}{ Feedback Rod Set 2} \\
\hline 1 & aluminum & 0.50 & 0.010 & 0.105 & 0.026 & 0.009 & 0.013 \\
\hline 2 & steel & 0.40 & 0.010 & 0.233 & 0.047 & 0.012 & 0.029 \\
\hline 3 & steel & 0.60 & 0.010 & 0.371 & 0.111 & 0.045 & 0.046 \\
\hline 4 & aluminum & 0.60 & 0.012 & 0.183 & 0.055 & 0.022 & 0.033 \\
\hline 5 & wood & 0.50 & 0.012 & 0.038 & 0.010 & 0.003 & 0.007 \\
\hline 6 & wood & 0.70 & 0.012 & 0.053 & 0.018 & 0.009 & 0.009 \\
\hline 7 & aluminum & 0.70 & 0.014 & 0.284 & 0.099 & 0.046 & 0.069 \\
\hline 8 & aluminum & 0.80 & 0.014 & 0.323 & 0.129 & 0.069 & 0.079 \\
\hline 9 & aluminum & 0.30 & 0.016 & 0.164 & 0.025 & 0.005 & 0.053 \\
\hline 10 & aluminum & 0.50 & 0.016 & 0.273 & 0.068 & 0.023 & 0.087 \\
\hline 11 & carbon & 0.40 & 0.020 & 0.050 & 0.010 & 0.003 & 0.043 \\
\hline 12 & carbon & 0.60 & 0.020 & 0.067 & 0.020 & 0.008 & 0.057 \\
\hline \multicolumn{8}{|c|}{ Test Rod Set } \\
\hline 1 & steel & 0.61 & 0.008 & 0.232 & 0.071 & 0.029 & 0.080 \\
\hline 2 & steel & 0.71 & 0.008 & 0.271 & 0.096 & 0.046 & 0.102 \\
\hline 3 & steel & 0.81 & 0.008 & 0.309 & 0.125 & 0.068 & 0.114 \\
\hline 4 & wood & 0.81 & 0.012 & 0.064 & 0.026 & 0.014 & 0.125 \\
\hline 5 & wood & 1.01 & 0.012 & 0.080 & 0.040 & 0.027 & 0.136 \\
\hline 6 & steel & 0.61 & 0.012 & 0.524 & 0.160 & 0.065 & 0.158 \\
\hline 7 & steel & 0.71 & 0.012 & 0.610 & 0.217 & 0.102 & 0.094 \\
\hline 8 & aluminum & 0.91 & 0.016 & 0.494 & 0.225 & 0.136 & 0.110 \\
\hline 9 & carbon & 0.71 & 0.020 & 0.093 & 0.033 & 0.016 & 0.012 \\
\hline 10 & carbon & 0.91 & 0.020 & 0.119 & 0.054 & 0.033 & 0.014 \\
\hline 11 & carbon & 1.01 & 0.020 & 0.132 & 0.067 & 0.045 & 0.019 \\
\hline 12 & carbon & 1.11 & 0.020 & 0.145 & 0.081 & 0.060 & 0.022 \\
\hline 13 & carbon & 1.21 & 0.020 & 0.158 & 0.096 & 0.077 & 0.025 \\
\hline
\end{tabular}

(Manuscript received April 2, 2008;

revision accepted for publication July 10, 2008.) 